\title{
A role for sodium channels in regulation of macrophage- mediated pathology in multiple sclerosis lesions
}

In a recent study of brain lesions obtained from patients with multiple sclerosis (MS), researchers have identified a role for the sodium channel $\mathrm{Na}_{\mathrm{v}} 1.5$ in the phagocytic pathway of myelin degradation by macrophages. Blockade of sodium channels could, therefore, represent a novel therapeutic strategy to attenuate macrophage-mediated CNS destruction. Joel Black and Stephen Waxman, lead investigators of the study published in the Multiple Sclerosis Journal, had previously demonstrated the expression of sodium channels by immune cells in the brain, including macrophages and microglia, but most of this work was performed in vitro. "It was important for us to examine the expression of sodium channels in situ within tissue obtained from the rapid autopsy of patients with MS, which were provided by our colleague Jia Newcombe," says Black.

The various compartments of the degradative pathway in macrophages

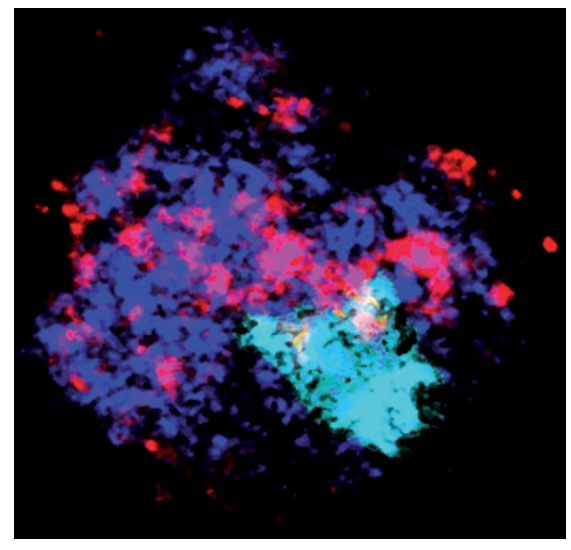

Triple staining of an acute multiple sclerosis plaque showing $\mathrm{Na}_{1} 1.5$ (red) expression in the late endosomes of macrophages - cells that are identifed by staining for HLA-DR (blue) and the nucleus (cyan). Image courtesy of J. Black.

(namely, phagosomes, early endosomes and late endosomes) were identified in brain samples from patients with secondary progressive MS through an immunohistochemistry-based approach. Co-staining for these macrophage structures and sodium channel isoforms revealed that $\mathrm{Na}_{\mathrm{v}} 1.5$ primarily localized to late (degradative) endosomes and those that contained myelin. Notably, $\mathrm{Na}_{\mathrm{v}} 1.5$ expression in late endosomes in the brain was evident solely in active MS lesions.

The findings, in the context of previous in vitro studies, suggest that the expression of sodium channels has a central role in the regulation of tissue destruction in MS lesions. "Our work provides a rationale for the development of targeted genesilencing of sodium channels to regulate the behaviour of inflammatory cells in MS," concludes Black. "This would be a novel approach, and is obviously a gamble, but it is a therapeutic avenue that we believe should be pursued."

Katy Malpass

Original article Black, J. A., Newcombe, J. \& Waxman, S. G. Nav1.5 sodium channels in macrophages in multiple sclerosis lesions. Mult. Scler. doi:10.1177/ 1352458512460417 